\title{
An Optimal Traffic Control Algorithm for 4G LTE Systems
}

\author{
Xinjian Cao ${ }^{1}$ and Rui Wang ${ }^{2 *}$ \\ ${ }^{1}$ Postgraduate office, Training department, Naval University of Engineering, Wuhan, Hubei, China \\ ${ }^{2}$ University library, Training department, Naval University of Engineering, Wuhan, Hubei, China
}

\begin{abstract}
The proper design of the 4G LTE network, in order to provide sufficient capacity of high-quality coverage and efficient terminal traffic management, is of utmost importance. Therefore, the joint LTE wireless communications network design problem is modeled in this paper as an optimization problem. The mathematical programming problem in the model is a nonlinear, non-convex, mixed integer-programming problem. Lagrangean relaxation and subgradient method are adopted to solve those complex problems because Lagrangean relaxation is a powerful mathematical tool to solve large-scale linear and non-linear programming problems. In the computational results, the optimal algorithm achieves more improvements compared to other primal heuristics, which could demonstrate the effectiveness of Lagrangean approach.
\end{abstract}

Keywords-orthogonal frequency division multiplexing; traffic control; optimal algorithm; Long term evolution

\section{INTRODUCTION}

In recent years there has been a huge increase in the demand for wireless communications, and the number of wireless subscribers will continue to grow. The overall trend is for the cellular phone to be preferred means of communication for the population. The requirement for cellular to expand its services beyond voice to multimedia and high-data-rate application has promoted the inception of a global Fourth generation standard.

The frequency reuse concept guides the cellular system design. The key elements in design cellular system include Cochannel Interference Reduction Factor (CIRF), Handoffs, Frequency Management and Frequency Assignment, ReverseLink Power Control, Capacity Enhancement, Orthogonal Frequency Division Multiplexing [1, 2]. However, traditional design rules for cellular networks are not directly applicable to LTE networks where intercell interference is not mitigated by cell placement and careful frequency planning.

In multi-cell LTE cellular networks, due to power control to alleviate near-far problem, a small cell adjacent to a large cell will experience a great deal of interference - which causes a significant reduction in the capacity of the small cell. We hope to adjust some effective control parameters which the network administrator can use to flexible distribute capacity in a multi-cell LTE network [3, 4]. Total call blocking rate is one of the most important measurement of the user's viewpoint to judge the subscriber. By adjusting the system parameters, we plan an integrated strategy to enhance the system usage for a long period. To fulfill the timing and the quality of the optimal decision, we construct a mathematical formulation that enhance twice or more times performance and cost improvement than general methods. Lagrangean relaxation has been proved that is good for solving the complicated mathematical mode, so we chose it as our tool for our problems.

\section{PROBLEM FORMULATION}

\section{A. Problem Description}

Total call blocking rate is one of the most important measurement of the user's viewpoint to judge the subscriber. By adjusting the system parameters, we plan an integrated strategy to enhance the system usage for a long period. In this long-term model, our objective is to minimize the call blocking rate of the network. In other words, the objective of this model is how to minimize total blocked traffic. When given: Candidate base station (BS) location and the distribution of users, traffic demand of each mobile terminal. The objective is to minimize the total blocked traffic. We should determine: Users' homing status, Radius of each base station (BS) and Power compensation factor. The constraints include: QoS constraint, capacity constraint, power compensation constraint, orthorgonality constraint. The assumption includes: Perfect power control is assumed, the reverse link is perfectly separated from the forward link, Multipath Fading is not considered, and User movement is not considered.

A legend of the notation used in the proposed mathematical formulation is given as follows. $G$ denotes the processing gain in the bandwidth state $i, W$ denotes total bandwidth, $K$ denotes data bit rate, $E_{b}$ denotes the energy BS received, $N_{0}$ denotes the background noise, $N_{\text {total }}$, denotes total noise, $\alpha$ denotes voice activity, $S$ denotes The power that a base station received from the mobile terminal that is homing to it with perfect power control, $B$ denotes the set of BSs, $T$ denotes the set of mobile terminals, $M_{j}$ denotes upper bound on number of users that can be handled by base station $j, \tau$ denotes attenuation factor, $D_{j t}$, denotes distance between base station $j$ and mobile terminal $t$, $D_{j j}$, denotes distance between base station $j$ and $j$ ', $I_{j}$, denotes the intracell interference, $I_{i j}$, denotes the interference from base station $j$ ' to $j, R_{j}$ denotes upper bound of radius of base station $j, \quad \lambda_{t}$ denotes traffic demand of mobile station t, $B\left(g_{j}, c_{j}\right)$ denotes Erlang B's Formula - Call blocking probability of traffic demand $g_{j}$ with available channels $c_{j}$, $b_{j}$ denotes call blocking probability of cell $j, \psi\left(n_{j}\right)$ denotes in-cell orthogonally factor, $\xi\left(n_{j}\right)$ denotes out-cell orthogonally factor, 
$\varepsilon$ denotes a small number, $\kappa$ denotes the minimal downlink power requirement, $\left(\frac{E_{b}}{N_{\text {total }}}\right)_{R L}$ denotes the minimum reverse link traffic channel SIR requirement $\left(\frac{E_{b}}{N_{\text {total }}}\right)_{F L}$ denotes the minimum foward link traffic channel SIR requirement.

The decision variables are given as follows. $\hat{c}_{j}$ denotes the number of users who can be active at the same time in the base station $j, r_{j}$ denotes transmission radius of base station $j, g_{j}$ denotes aggregate flow in base station $j$ (in Erlangs), $z_{j t}$ denotes decision variable which is 1 if mobile terminal $t$ is serviced by base station $j$ and 0 otherwise, $\beta_{j}$ denotes power compensation factor of cell $j$.

\section{B. Problem Formulation}

The following is the problem formulation of the optimization problem, and we will make some descriptions below:

Objective function is as below:

$$
Z_{I P}=\min \sum_{j \in B} g_{j} B\left(g_{j}, \hat{c}_{j}\right)
$$

subject to:

$$
\begin{aligned}
& \left(\frac{E_{b}}{N_{\text {total }}}\right)_{R L} \leq \beta_{j} \frac{S}{N_{0}} /(1+ \\
& \frac{1}{G} \alpha \frac{S}{N_{0}} \beta_{j}\left(\hat{c}_{j}-1\right) \psi\left(\hat{c}_{j}\right)+ \\
& \left.\frac{1}{G} \alpha \frac{S}{N_{0}} \sum_{\substack{j, j \in B \\
j, \neq j}} \beta_{j},\left(\frac{r_{j},}{D_{j j^{\prime}}-r_{j^{\prime}}}\right)^{\tau} \psi\left(\hat{c}_{j},\right) \hat{c}_{j}\right) \\
& \left(\frac{E_{b}}{N_{\text {total }}}\right)_{F L} \leq \frac{\frac{\kappa}{N_{0}}}{1+\frac{1}{G} \alpha \frac{\kappa}{N_{0}}(1-\rho)+\frac{1}{G} \alpha \frac{\kappa}{N_{0}} \sum_{\substack{j^{\prime} \in B \\
j^{\prime} \neq j}}\left(\frac{D_{j j^{\prime}}}{r_{j}}\right)^{-\tau}} \\
& 1 \leq \beta_{j} \leq \bar{\beta}_{j} \forall j \in B \\
& B\left(g_{j}, \hat{c}_{j}\right) \leq b_{j} \forall j \in B \\
& g_{j}=\sum_{t \in T} z_{j t} \lambda_{t} \forall j \in B \\
& D_{j t} z_{j t} \leq r_{j} \forall j \in B, t \in T
\end{aligned}
$$

$$
\begin{aligned}
& D_{j j^{\prime}} \geq \operatorname{Max}\left(r_{j}, r_{j^{\prime}}\right)+\varepsilon \quad \forall j, j^{\prime} \in B \\
& 0 \leq r_{j} \leq R_{j} \forall j \in B \\
& \sum_{j \in B} z_{j t}=1 \forall t \in T \\
& \hat{c}_{j} \leq M_{j} \forall j \in B \\
& z_{j t}=0 \text { or } 1 \forall j \in B, t \in T
\end{aligned}
$$

The objective function is to minimize the total blocked traffic of the system after it has been operated.

Constraint (1) is to ensure that each traffic source is served with its base station with required QoS (Reverse link) [5]. The left hand side of this inequality is the minimal SIR which each connection should be hold. The right hand side means the real SIR. The denominator of the right hand side is the total interference value, including white noise, the intra-cell interference[6], and inter-cell interference[7]. Additionally we introduce power compensation factor to enhance our objective. Constraint (2) is to ensure that each traffic source is served with its base station with required QoS (Forward link). The left hand side of this inequality is the minimal SIR which each connection should be hold. The right hand side means the real SIR. The denominator of the right hand side is the total interference value, including white noise, the intra-cell interference, and inter-cell interference. Constraint (3) denotes power compensation factor is bounded between 1 and maximal power compensation of each base station. Constraint (4) is to ensure that any base station can serve its slave mobile terminal under certain call blocking rate. Constraint (5) is to ensure that if a base station does not provide service to a mobile terminal, then the decision variable $z_{j t}$ must equal to 0 . Constraint (6) is to ensure that a base station can only serve the mobile terminals that are inside its coverage area of effective radius. Constraint (7) is to ensure the distance between two base stations is larger than any effective radius of each one. Constraint (8) is to ensure that the transmission radius of each base station ranges between 0 and $R_{j}$. Constraint (9) is to ensure that each mobile terminal can be homed to only one base station. Constraint (10) is to ensure that the number of users who can be active at the same time in a base station would not exceed the base station's upper bound. Constraint (11) is to enforce the integer property of the decision variables.

\section{SOLUTION APPROACH}

\section{A. Lagrangean Relaxation Based Algorithm}

The basic approach to the development of the solution procedure to Formulation (IP) is Lagrangean relaxation. Lagrangean relaxation is a method for obtaining lower bounds for minimization problems as well as good primal solutions in 
integer programming problems. The main steps of Lagrangean relaxation method are as following: relax complicating constraints, multiple the constraints relaxed with corresponded Lagrangean multipliers, and add them to the primal objective function. Decompose the problem into several independent subproblems that could be optimally solved. Then solve them optimally and get Lagrangean dual problem solution. By using the Lagrangean relaxation method, we can transform the primal problem (IP) into the following Lagrangean relaxation problem (LR) where Constraints (1), (2), (5), and (6) are relaxed.

The algorithm is used to deal with the Model We call it LR. Shown as follow:

Step1: Initialize network topology parameter.

Step 2: Calculate constant parameters, such as $\mu_{j t}, D_{j t}$ and assign Lagrangean relaxation improve counter to equal 50.

Step 3: Initialize multipliers.

Step 4: According to the given multipliers, optimally solve these problems to get the value of $Z_{\text {dual }}$.

Step 5: According to heuristics mentioned in next section (Adjusting Procedures), get the total call blocking traffic, the value of $Z_{I P}$.

Step 6: If $Z_{I P}$ is smaller than $Z_{I P} *$, we assign $Z_{I P} *$ to equal $Z_{I P}$. Otherwise, we minus 1 from the improvement counter.

Step 7: Calculate step size and adjust Lagrangean relaxation multipliers.

Step 8: Iteration counter increases 1 . If interaction counter is over threshold of system, stop this program. And, $Z_{I P} *$ is our best solution. Otherwise, repeat Step 4.

\section{B. Adjusting Procedures}

The initial plan gotten from Algorithm above and Lagrangean results will be fed into an adjusting procedure to get feasible solutions to primal problem. The procedure includes 3 stages: (1) get feasible solution under the allfunctional state, (2) consider all possible failure scenario to meet the coverage ratio requirements, QoS requirement, and outage probability constraint, (3) channel assignment.

Lagrangean results will be examined and adjusted. Because of the complexity of coupling of decision variables in this problem, we have to exhaustively search all the possible solution sets of decision variables. Fortunately, the value of the radius power, $r_{j}$, and the capacity, $\hat{c}_{j}$, defined in this problem are discrete and limited. Somehow, the power compensation factor $\beta_{j}$ is continuous decision variable. So we can begin dealing with the radius power in each getting primal feasible solution iterations. Since the radius power was determined, we can calculate the aggregate traffic, $g_{j}$ of every base station under its radius power currently. Of course, the capacity and QoS constraints described in previous section must be held. And under a pair of $\left(g_{j}, \hat{c}_{j}\right)$, the call blocking probability has to be lower than a desired level, say, $1 \%$ or lower. The heuristic used in this problem is described in the following.
Step 1: Use Lagrangean results $r_{j}$ and $\beta_{j}$ as an initial arrangement of base stations. Compute number of mobile stations within $r_{j}$. Sort base stations by the value and assign mobile stations to them according to this order until the capacity is used out. If the plan is feasible, terminate.

Step 2: For each base station $j$ that violates the QoS constraint, sort multipliers we got from $Z_{\text {dual }}$ in decreasing order respectively. Select base stations to adjust in this order. Then, for each base station $j$, we compare $v_{j}^{1}$ with $v_{j}^{2}$. If $v_{j}^{1} \geq v_{j}^{2}$, we try to adjust power compensation factor $\beta_{j}$ until the QoS constraint (1)(2) is held. If $v_{j}^{1}<v_{j}^{2}$, we try to decrease down the radius power first. Then we construct a reasonable draft plan.

Step 3: Then, we check the call blocking probability constraint. If it is originally satisfied, going to step 4; otherwise we start try to re-homing the farthest traffic source until the call blocking rate is under a desired level.

Step 4: Since the radius power $r_{j}$ of each base station was determined. We can calculate the aggregate traffic of each base station.

Step 5: For each base station $j$, we find out the largest distance between any traffic source and base station. Try to narrow the radius power down.

Step 6: If base station still can provide service to the largest traffic source, we just set the new value to $r_{j}$; otherwise retain the original status of $r_{j}$.

Step 7: Finally, according to the set of decision variables we process in previous steps, we can calculate the actual traffic that is blocked by the system.

\section{COMPUTATIONAL EXPERIMENTS}

The following experiments would like to show the effectiveness of Lagrangean results compared with other primal approaches. The approach is to compare the initial plans produced by Lagrangean and primal heuristics. One reasonable general primal heuristic is proposed in Algorithm 3.1.

The Assumptions are: Calls arrive according to a Poisson process [8], the demand is randomly distributed in the area, 10 randomly generated examples of user distribution are tested in the experiment, and the mobility of mobile terminals is not our concern.

According to reference work [9], the parameters are defined as below. $S / N_{0}=7 \mathrm{db}, E_{b} / N_{\text {total }}=6.58 \mathrm{db}$, Call blocking probability of links $=1 \%, 250$ traffic sources, Traffic demand per traffic source $=1.1$ erlangs, Call blocking probability $=1 \%$, Processing Gain=520.8333 (5 MHz/9.6 Kbps), Voice Activity factor $=0.375$, Service area $=400 \mathrm{~km}^{2}$, Maximum number of channels that can be assigned to a base station $=120$, Possible power radius of base stations: $1000 \mathrm{~m} \sim 4000 \mathrm{~m}$, The detailed configurations of candidate locations are then described in the next section. 


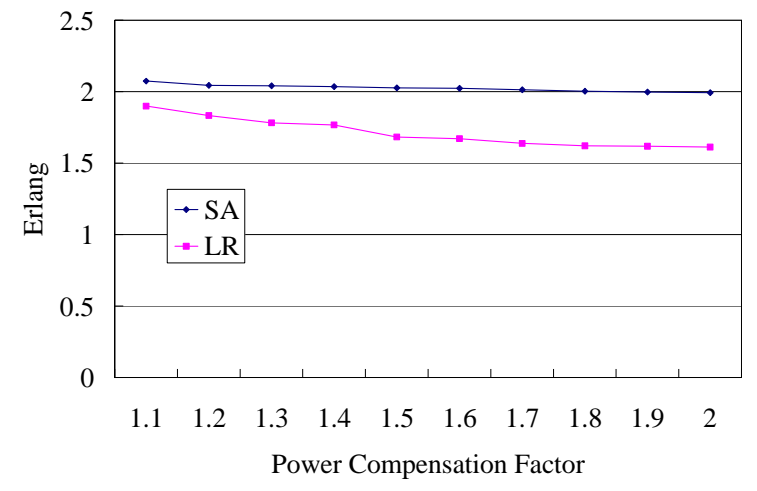

FIGURE I. THE COMPARISON OF SA AND LR WITH FIXED POWER COMPENSATION FACTOR ADJUSTMENT

We set all BSs' power compensation factor all the same range from 1.0 to 2.0 and test in 10 different cases with different user distribution which all are random uniform distributed. We present the experiment results in the average of 10 different cases for convenience as shown in Figure 1. When we increase the power, the system total call blocking rate will decrease. However, the decrease is not much.

We freely adjust each BSs' power compensation factor range from 1.0 to 2.0 and test in 10 different cases with different user distribution which all are random uniform distributed. The Lagrangean-based heuristic and the primal one are named "HR" and "PO", respectively. PO_Beta and HR_Beta denote the results with variable power compensation factor adjustment in each base station respectively. The experiment result shows the effect of power compensation factor adjustment is amazing as shown in Figure 2. The improvement $\left(=\frac{L R-L R_{-} \text {Beta }}{\text { LR_Beta }}\right)$ is more than 10 times.

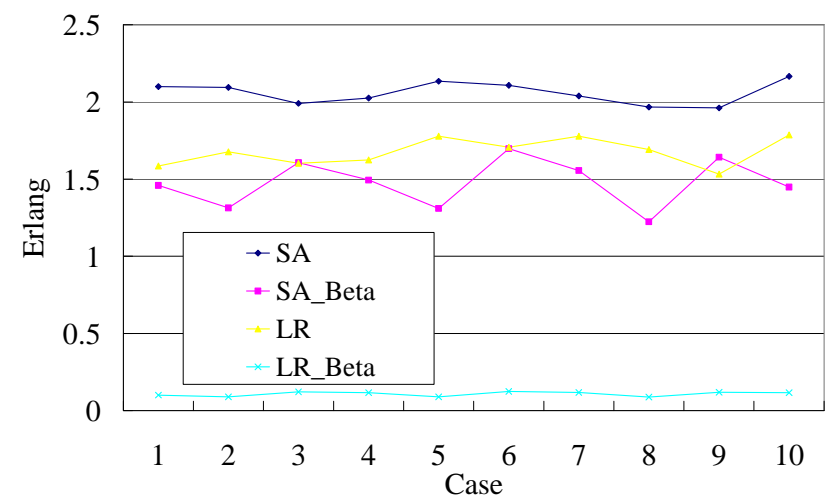

FIGURE II. THE COMPARISON OF PO, PO_BETA, HR, AND HR_BETA WITH PCF ADJUSTMENT VS WITHOUT PCF ADJUSTMENT

In the third case, we try to present the effect of the increase of traffic load. We increase the traffic demand per traffic source from 1.1 to 2.2. We freely adjust each BSs' power compensation factor range from 1.0 to 2.0 and test in 10 different cases with different user distribution which all are random uniform distributed.

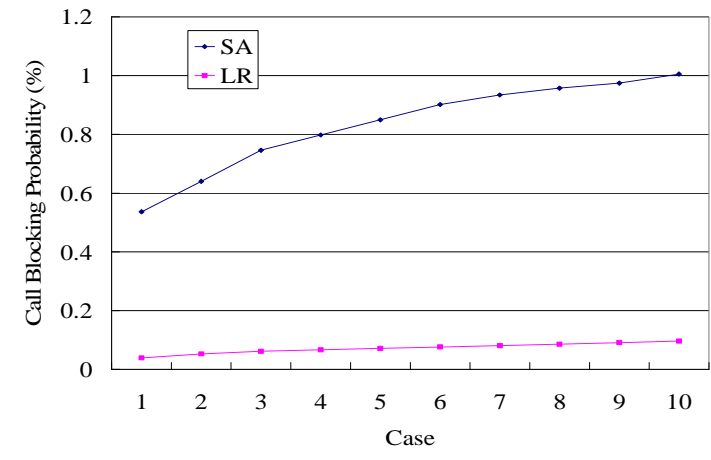

FIGURE III. THE AVERAGE CALL BLOCKING PROBABILITY COMPARISON OF PO AND HR WITH DIFFERENT TRAFFIC LOAD

We can see that total call blocking rate and average call blocking probability both increase as the traffic load increase as shown in Figure 3. However, our Lagrangean-based heuristic provides an effective approach to achieve our requirement.

In our computational results, our Lagrangean relaxation based algorithms achieve up to $24.61 \%$ and $20.30 \%$ improvements compared to other primal heuristics in the case 10 and 18 candidate nodes are considered, which could demonstrate the effectiveness of Lagrangean approach.

\section{CONCLUSION}

We present an approach to design and manage a LTE wireless communication network. We can express our achievements in terms of formulation and performance. In terms of formulation, we model a mathematical expression to describe the overall LTE wireless communication network design and management problem. We consider both nonregular size cell and non-uniform traffic demand to make this thesis more generic. Due to the complexity of this problem, we pick Lagrangean relaxation and subgradient method as our main methodology. When using these mathematical tools, they can provide us some hints to improve our heuristics. In terms of performance, our Lagrangean relaxation based solution has more significant improvement than other intentional algorithm.

\section{REFERENCES}

[1] S. B.Rejeb, N.Nasser, S.Tabbane, "A novel resource allocation scheme for LTE network in the presence of mobility,” Journal of Network and Computer Applications, vol.46, 2014, pp. 352-361.

[2] L. Mogan, V. Mantharachalam, G. Kalaichelvi, et al. "Dynamic Markov Model based spectrum sharing and band aggregation for LTE-700 MHz band in India,” Proc. 2015 3rd International Conference on. IEEE Signal Processing, Communication and Networking (ICSCN), 2015, pp. 1-6.

[3] N. A. Amirrudin, S. H.Ariffin, N. Malik, "Mobility prediction via Markov model in LTE femtocell," Internation Journal of Computer Application, vol. 65, 2013, pp. 40-44.

[4] X.Xiang, C.Lin, X.Chen, et al. "Toward optimal admission control and resource allocation for LTE-A femtocell uplink,” IEEE Transactions on Vehicular Technology, vol. 64 2015, pp. 3247-3261.

[5] K. B.Ali, M. S.Obaidat, F.Zarai, et al. "Markov model-based adaptive CAC scheme for 3GPP LTE femtocell networks," 2015 IEEE International Conference on Communications (ICC). IEEE, 2015, pp. 6924-6928. 
[6] T.Tsang, M.Bilal, P. M. Chan, et al. "Performance Analysis for LTE Networks with Markov Decision Process," Cyber Journals: Multidisciplinary Journals in Science and Technology, Journal of Selected Areas in Telecommunications, August Edition, vol. 3, 2013, pp. $1-10$.

[7] W. Kim, G.S. Park, H. Song, "An effective cross-layer designed packet scheduling, call admission control, and handover system for video streaming services over LTE network," Journal of Visual Communication and Image Representation, vol. 31, pp. 335-346, March 2015.

[8] V.Y. Borodakiy, K.E. Samouylov, I.A. Gudkova, "Analyzing Mean Bit Rate of Multicast Video Conference in LTE Network with Adaptive Radio Admission Control Scheme,” Journal of Mathematical Sciences, vol. 218, pp. 257-268, July 2016.

[9] J. Zhao, TQS Quek, Z. Le, "Heterogeneous Cellular Networks Using Wireless Backhaul: Fast Admission Control and Large System Analysis, "IEEE Journal on Selected Areas in Communications, vol. 33, pp.2128 -2143 , June 2015 